\title{
Referee Acknowledgement for 2016
}

In this issue, we publish the names of those who reviewed manuscripts for us in 2016.

The Editor-in-Chief, Specialist Editors and everyone involved in publishing BJC would like to extend our sincere thanks to the referees for contributing their expertise and time. Our referees play an invaluable role ensuring that $B J C$ continues to publish the high-quality original papers and reviews that make it one of the world's leading oncology journals.

Tarek MA Abdel-Fatah

Anya Adair

Rosalind Adam

David Adams

Peter Adams

Richard Adams

Douglas James Alexander Adamson

Christina L Addison

Olivier Adotevi

Thomas Ahern

Nihal Ahmad

Erin J Aiello Bowles

Jaffer A Ajani

Thankamma Ajithkumar

Agneta Akesson

Suminori Akiba

Khawla S Al-Kuraya

Emilio Alba

Peter C Albertsen

Leo Alexandre

James Allan

Maria Alsina

Maarten Altelaar

Alberto Amadori

Frederic Amant

Carlos Ivan Amaya-Chanaga

Maria R Ambrosio

Eitan Amir

Roland A Ammann

Christopher I Amos

Jesus Anampa

Joseph C Anderson

Therese M-L Andersson

Yvette Andersson

Jeffrey Andolina

Gabriella Andreotti

Ritu Aneja

Christina M Annunziata

Alessandro Antonelli

Giuseppe Aprile

Walter Arancio

Marc Arbyn

Alexandre Arcaro
Marco Archetti

Hendrik-Tobias Arkenau

Gregory T Armstrong

Melina Arnold

Rudolf Arnold

Igor Astsaturov

Andische Attarbasch

Gerhardt Attard

Laura D Attardi

Josep M Auge

Helmut G Augustin

Philippe Autier

Anssi Auvinen

Adnan Aydiner

Ninel Azoitei

Hideo Baba

Jean-Baptiste Bachet

Brett Bade

Sunil Badve

Jeong Mo Bae

Catherine Bagot

Shohbit Baijal

Stuart G Baker

Shairaz Baksh

Christopher Baliski

Stuart Ballantyne

Katarina A Balter

Udai Banerji

Emily Banks

Finian J Bannon

Stephen Barclay

Agueda Martinez Bariocanal

Jill S Barnholtz-Sloan

MP Barr

Sophie V Barrett

Jorge Barriuso

Thomas F Barth

David Basanta

Adam Bass

Bristi Basu

Luc Bauchet

Michael Baum

Daniel Baumhoer
Daniel Beachler

Emma Beddowes

Alicia Beeghly-Fadiel

Tomasz M Beer

Elizabeth A Beierle

Klaus Beiske

Tanios Bekaii-Saab

Sara Benitez-Majano

Al B Benson

Sally Claire Benton

Sébastien Benzekry

Francesca Beretti

Anna Berg

G Berg-Beckhoff

Barry M Berger

Daniel M Berney

Francesco Bertolini

Eric M Bessell

Johannes Betge

Chetan Bettegowda

Dominik Bettinger

Benoit Beuselinck

Charlotte Bevan

Angelika Bezan

Aashish Bhatt

Moshmi Bhattacharya

Ujjal Kumar Bhawal

Ettore Biagi

Fabrizio Bianchi

Andrew V Biankin

Nicoletta Biglia

Eliana Bignotti

Kristin Bixel

Niclas Björn

Jon K Bjerregaard

Sarah P Blagden

Jeremy Blaydes

Maria Blettner

Prunella Blinman

C Bodelon

Stefan Boeck

Maximilian Boesch

Paolo Boffetta
AD Boiko

Efren Bolivar

Stefano Bonassi

Carolina Bonilla

Petri Bono

Stefanos Bonovas

Arnaud Boot

Christopher M Booth

Ivan Borbath

Michael Bordonaro

Hossein Borghaei

Signe Borgquist

Annabel Catherine Borley

David W Borowski

Marilyn J Borugian

Amanda Bos

Marcus Bosenberg

Cristina Bosetti

F T Bosman

Dominick Bosse

Yves Boucher

François Boudreau

Ioannis Boukovinas

Anne Bowcock

Chiara Braconi

Patrick T Bradshaw

Kate E Brain

Michael Graham Bramble

Jacopo Branca

Judith Brand

Donal J Brennan

Darren R Brenner

Jerry Brewer

David H Brewster

John Bridgewater

Louise A Brinton

John Brodersen

Andrew S Brohl

Agnieszka Bronisz

Ewan Brown

Jessica Brown

Robert Brown

Kristoffer Brudvik 
Adam Brufsky

James Brugarolas

Matteo Brunelli

Daniel Brungs

Ulrike Bruning

Eleonora Bruno

Elizabeth Susan Buckley

Alfredo Budillon

Mary Ann Burg

Maximilian Burger

Bryan Burmeister

Eric Burton

Theresa M Busch

Elisa Caffarelli

Caitriona Cahir

Qiuyin Cai

Francesco Caiazza

John Cairns

George Calin

David Cameron

Ana Paula Campanelli

Jared M Campbell

Stewart Campbell

Elias Campo

Silvana Canevari

Amparo Cano

Robert Canter

Yin Cao

Rocco Cappellesso

Vera Cappelletti

Michele Carbone

Chris R Cardwell

Matteo S Carlino

Arkaitz Carracedo

Jason Carroll

Ross Carruthers

David Raul Francisco Carter

Andrea Casadei Gardini

Oriol Casanovas

James Cassidy

Philippe A Cassier

Sergi Castellvi-Bel

Juan P Cata

Vincenzo Catalano

Richard Cathomas

Emma Cattell

Wim P Ceelen

Young Kwang Chae

Anthony J Chalmers

Ann F Chambers

Hsiao Chang Chan

Stephen L Chan

NS Chandel

Dhyan Chandra

David Chang

Kai-Ping Chang

Yeun-Chung Chang

Stephen J Chanock

Dana M Chase

Etienne Chatelut

Suman Chatterjee

Balkrishna Chaube

Mariana Chavez-MacGregor

Bin Chen

D Chen

Fei Chen

Ronald Chen

Sidi Chen

Karis Kin-fong Cheng

Q Cheng
Shi-Yuan Cheng

Ravindresh Chhabra

Stephen K Chia

Johanna Chiche

E Gabriela Chiorean

Chao-Hua Chiu

Alexandre Chlenski

Jeong Hee Cho

Chel Hun Choi

Joon Young Choi

Woonyoung Choi

Alan Christie

Shu-Chun Chuang

Jonas Cicenas

Esther Una Cidon

Jean Clairambault

Jonathon Clark

Geoffrey J Clark

Paul A Clarke

Robert Clarke

Stephen Clarke

Michael Cleary

Nicholas J Clemons

Anne-Marie Cleton-Jansen

Alan Coates

Philip J Coates

Graham Colditz

Robert E Coleman

Marco Colleoni

Anne T Collins

Peter Collins

Fiona Collinson

Ada Collura

Danny Colombara

Alfonso Colombatti

Luciano Conti

Natalie Cook

Colin S Cooper

An Cooseman

Angel Corbi

Pippa G Corrie

JM Coulson

Nigel C Cowan

Joanne Crawford

Nigel Crawford

Jenette Creaney

Nicola Cresti

Canien Creutzberg

Alastair E Cribb

Deirdre P Cronin-Fenton

Tom Crosby

Margaret Cruickshank

A Cuenda

Long Cui

Eoin Cummins

David Cunningham

Giuseppe Curigliano

Anne E Cust

Jack Cuzick

Qi Dai

Y Dai

Luigino Dal Maso

Angus G Dalgleish

Sambasivarao Damaraju

Adam Dangoor

Sarah Danson

Emile Darai

Sarah Darby

Sarah C Darby

Bhudev C Das
Prajnan Das

A Dasari

Adil I Daud

Sarah E Daugherty

Jeff Davies

Bazbek Davletov

Shaheenah Dawood

Heather Dawson

Pradip De

Evandro de Azambuja

Jana de Boniface

Johann S de Bono

Marianna de Camargo Cancela

Ana de Carvalho

Harry J de Koning

Paul De Leyn

Mauro De Santi

Eduardo De Stefani

Olivier De Wever

Ronald de Wit

Ralph J DeBerardinis

Kathleen M Decker

Julie Decock

Suzette Delaloge

Dustin A Deming

David T Denhardt

Nicholas C Denko

Paul Dent

Carol DeSantis

Jérôme Devy

Nandini Dey

Punita Dhawan

Chiara Di Girolamo

Nick Di Girolamo

Massimo Di Maio

Antonio Di Meglio

Attilio Di Spiezio Sardo

Robert Diament

Don J Diamond

Luis A Diaz Jnr

Thomas Diepgen

Jayne Digby

Francoise Dignat-George

Joakim Dillner

Jeak Ling Ding

David Dingli

Gillian Dite

Antonio Ditto

Sanjay Dixit

Robert Doebele

Suhail Doi

Helmut Dolznig

Enric Domingo

Laia Domingo

Kristine A Donovan

Efrat Dotan

Amy Downing

Charles W Drescher

Yvette Drew

Timothy R Driscoll

Liat Drucker

Andreas du Bois

Zhenfeng Duan

Catherine Dube

Gillian M Duchesne

Michael J Duffy

Stephen W Duffy

Reinhard Dummer

Charles Dumontet

Laurie Dunn
Natalie Dupre

Nelson J Dusetti

Mark Duxbury

Martin Dyer

Julie Earl

Julien Edeline

Michael Edlinger

Dylan R Edwards

Thomas Efferth

Wafik S El-Deiry

Aristides G Eliopoulos

Peter Ellis

Zakaria S Eltahir

Peter Elwood

Jon D Emery

Ellen G Engelhardt

Monica Jernberg Engstrøm

Zandra Nymand Ennis

Meira Epplein

Jonathan I Epstein

Rune Erichsen

Antoine Eskander

Katharine Esselen

D Gareth Evans

Mark Francis Evans

Serdar Evman

Michael S Ewer

Marianne Ewertz

Roberto Fabiani

Ibrahim Fakhr

Carole Fakhry

Stephen J Falk

Ou Fang-Shu

Oliver Farges

Fabio Farinati

Jane Farmer

Susan Mary Farrington

Gelareh Farshid

Juliane Farthmann

Nicola Fazio

Moritz Felcht

Richard G Feltbower

Sarah Fendt

Karen S Fernandez

Pietro Ferrari

laura Ferris

Maria Feychting

Walter Fiedler

Stacey Finley

Gaetano Finocchiaro

Barbara J Fisher

Brian T Fisher

James M Flanagan

AD Flaxman

Gini Florence Fleming

Olivia Fletcher

Kean Florence

Cornelia Marlene Focke

Gerald Fogarty

Andrew Folpe

Gunnar Folprecht

Anders Fomsgaard

Vincenzo Formica

Gianni Forti

Joan Fortuny

David A Foster

George Fountzilas

Agnès Fournier

Jacques Fracheboud

Adele Margaret Francis 
Hester Franks

Christian Frezza

A Friedl

Eitan Friedman

Michael Froehner

Siqing $\mathrm{Fu}$

Naoya Fujita

Masahiro Fukuoka

Daniel Fults

Yasuhiro Funahashi

Helena Furberg

Jurgen J Futterer

Edward Gabrielson

Julia C Gage

G Gaidano

Kezia Gaitskell

Ramesh K Ganju

Patricia Ganz

AC Gao

Dingcheng Gao

Yuzhen Gao

Ingrig Garajova

Joel Garbow

Rocío García-Becerra

Maria Jose Garcia

Michela Garofalo

Federico Garrido

Maria Luisa Gasparri

Gagan Gautam

Anna T Gavin

Andrew Gaya

Mark Gaze

Richard Gelber

David E Gerber

Marco Gerlinger

Paula Ghaneh

Hazem Ghebeh

Rita Ghosh

Giuseppe Giaccone

Riccardo Giampieri

Geoffrey Gibney

Gretchen L Gierach

Francesco Giganti

Yolanda Gilaberte

Duncan Charles Gilbert

Ethel S Gilbert

Clare Gilham

Blake Gilks

Roopinder Gillmore

Ophira Ginsburg

Paolo Giorgi Rossi

Elisa Giovannetti

Meritxell Gironella

Dimitra Gkika

Stan Glantz

Hilary Glen

Paul Glen

Bengt Glimelius

Catherine Glynn

Rob Glynne-Jones

Michael Gnant

Valentin Goede

James J Going

Talia Golan

James R Goldenring

Tamas Gonda

Paul Goodfellow

Kylie Gorringe

Hadi A Goubran

Christine Grady
Inger Gram

Michael Graner

Edward Graves

Alastair M Gray

Natallia Gray

Daniel M Green

Alastair Greystoke

Wolfgang Grisold

Bjorn Henning Gronberg

Viktor Gruenwald

Jian $\mathrm{Gu}$

Henk-Jan Guchelaar

Richard Guerrant

Rachel Guest

Robert Gunier

Qi Guo

Yubin Guo

Avinash Gupta

Girish Gupta

Rishein Gupta

Maha Gurumurthy

Kilian Martin Gust

Ana Gutiérrez-Fernández

Alejandro Gutierrez

Michael Haas

Ulrich Thorsten Hacker

Angela Hague

Jorg Haier

Reza Hakkak

Per Hall

Daniel G Haller

Chris Halsey

Dolores Hambardzumyan

William T Hamilton

Pascal Hammel

Ester M Hammond

Jiali Han

Ze-Guang Han

Philip C Hannaford

Jose AU Hardillo

B Haring

Adrian L Harris

Holly Harris

Samantha Leigh Harrison

Christopher D Hart

Helene Hartvedt Grytli

Kamrul Hasan

Henrik Hasle

J A Hassell

Maureen Hatch

Mickael Hauptman

Maria A Hawkins

Robert E Hawkins

Nicholas Hayward

Biao He

Jing $\mathrm{He}$

Ping $\mathrm{He}$

Susan Heavey

Hannelore Heemers

Christopher Heeschen

Nancy Heffernan

Balazs Hegedus

Matthew D Hellman

Andrew E Hendifar

Sean Hennessy

Luis Alberto Henríquez-Hernández

Paul Hewitson

Dominic Heymann

Ryan M Hickey

Theresa Hickey
Hideaki Shimada

Martin S Highley

Mark Hill

Michelle Hill

David Hilton

Masuda Hiroko

Anders Hjerpe

Fay J Hlubocky

Cheryl Ho

Daniel Hochhauser

Timothy Hoey

Gunther Hofbauer

Camilla Molich Hoff

Michael Hoffmeister

Thomas Hogberg

Marianne Hokland

Stefan Holdenrieder

Marina K Holz

Satoshi Honjo

Laura Horsley

Michael Robert Horsman

Anthony Howell

James J Hsieh

Chao-Yuan Huang

Tim Hui-Ming Huang

Joleen Hubbard

Robert A Huddart

Barry I Hudson

Florence Huguet

David Hui

Laetitia Huiart

Patrick Humbert

Amanda B Hummon

Paula Hurley

Adam Hurlstone

Syed A Hussain

Salman M Hyder

David Hyman

Shingo Ichimiya

Giandomenica Iezzi

Thomas Iftner

David H Ilson

Mohammad Ilyas

Stefan Indraccolo

Pasquale Fabio Innominato

Michael R Irwin

Tariq Ismail

Yoshifumi Itoh

Salma Jabbour

David Jackson

Rakesh Jain

Filip Janku

Wolfgang Janni

Tobias Janowitz

Lynn A Jansen

Ismail Jatoi

Gordon C Jayson

Michael Jefford

Valerie A Jenkins

Allan Jensen

Henry Jensen

Lars Henrik Jensen

Barbara A Jereczek-Fossa

Helena Jernstrom

Carmen Jeronimo

Jose A Jeronimo

BC Jham

Jianguang Ji

Hongbing Jiang

Zhen Jiang
Li Jiao

Renjie Jin

Karin Jirstrom

Heikki Joensuu

Markus Joerger

Christoffer Johansen

Mattias Johansson

Kenneth C Johnson

Peter WM Johnson

Daniel Joseph Jones

Philip H Jones

Robert $\mathrm{H}$ Jones

Terry M Jones

Claus Jorgensen

Doireann Patricia Joyce

Przemyslaw Juszcznski

Martin Köbel

Yoshihiro Kakeji

Virginia Kaklamani

Thomas Kalinski

Tuula Kallunki

Tania Kalsi

Jurre Jorian Kamphorst

Tatsuya Kanto

Sonja Kappel-Latif

Giorgos Karakousis

Dimitrios Kardamakis

Susan Kasper

Masaru Katoh

Charles Keller

Charles Kelly

Karl T Kelsey

Nancy E Kemeny

Thomas W Kensler

David Kerr

Santosh Kesari

Ralf Ketter

$\mathrm{NaNa}$ Keum

Nigel Key

Timothy J Key

Yasmin Khakoo

Saad Khan

K Khazaie

Ivana Kholova

Syed Ali Khurram

Nikhil I Khushalani

Kostatinos Kiakos

Jason M Kidd

Michael K Kiessling

Annamari Kilkkinen

Anne E Kiltie

Dong-Wan Kim

Isaac Yi Kim

Wun-Jae Kim

Randall J Kimple

Mark O Kitchen

Tohru Kiyono

Naomi Kiyota

Tobias Klatte

Sonja Klebe

Jorg Kleeff

Adi Klil-Drori

Jennifer Knox

Andrew H Ko

Aung Ko Win

Hemant M Kocher

Alison B Kohan

Elise C Kohn

Meri Koivusalo

Karin Stinesen Kollberg 
Toshikazu Kondo

Gottfried E Konecny

Badrinath R Konety

David Kooby

Marcel Kool

Michael I Koukourakis

Anita Koushik

Magdalena Koziol

Robert Arthur Kratzke

Mechthild Krause

Clemens Krepler

Andreas Krieg

Mieke Kriege

Rebecca Kristeleit

Nils Kroeger

Henry Mark Kuerer

Walther Kuhn

Ernst Kuipers

Deepak Kumar

Ashok Kumar Pandurangan

Pamela Kunz

Volker Kunzmann

John S Kuo

Sung-Hsin Kuo

Emila Kurbasic

Shigeki Kusamura

Heidi N Kusters-Vandevelde

Natasha Kyprianou

Stefan Lönnberg

Caterina AM La Porta

Carlo La Vecchia

Satyanarayana Labani

Francine Laden

Gabriel Y Lai

H-C Lai

Anni Laine

Barry J Laird

Angela Lamarca

Paul C Lambert

Samy Lamouille

Maria Grazia Lampugnani

Santo Landolfo

Simon P Langdon

Thorsten Langer

Ruth E Langley

Cord Langner

Norm Laperriere

James M Larkin

Anders Christian Larsen

L Larue

Ulrik Lassen

Silke Lassmann

Dominique Laurier

Alessandro Laviano

Sean E Lawler

James S Lawson

Alexander Lazar

Luca Lazzari

Dung T Le

John Le Quesne

Christophe Le Tourneau

Michael Leahy

Valerie LeBleu

Andre Lechel

Jonathan A Ledermann

Jung-Min Lee

Marie Catherine Lee

Glen A Lehman

Lawrence Leichman

Alexander B Leichtle
Karin Leifland

Hannah Lennon

Guido Lenz

Bob Li

Wen-Qing Li

Wenliang Li

Xin Li

Yong $\mathrm{Li}$

Johanna Liebl

Gerrit-Jan Liefers

Douglas I Lin

J Lin

Jianqing Lin

Kangyu Lin

Ming-Tseh Lin

Patrick P Lin

Shiaw-Yih Lin

Zongming Lin

Spiros Linardopoulos

Michael Lind

Daniel J Lindner

David Lindquist

Aija Line

Martha Linet

Lance Liotta

Michael P Lisanti

Muriel Lize

Hans-Gustaf Ljunggren

Ana Lluch

Stacy Loeb

Fiona Lofts

Richard FA Logan

Martijn Lolkema

André Lollert

Juanita Lopez

I Lorand-Metze

Christopher J Lord

Simon Lord

Florian Lordick

Adriana Tarla Lorenzi

Jiade $\mathrm{Lu}$

Meixia Lu

Min $\mathrm{Lu}$

Zhen Lu

Claudio Luchini

Alessandro Lugli

Lars Lundell

Miguel-Angel Luque

Elsebeth Lynge

Georgios Lyratzopoulos

Antti Mäkitie

Henrik Møller

Wencai Ma

Yuk Ting Ma

Antonio Maccio

John R Mackey

Dan MacLean

Iain RJ Macpherson

Kelley Madden

Oliver Maddocks

Robert M Mader

Ayman Madi

Roberta Maestro

David Magne

Haider Mahdi

Rob Mairs

Michael Ben Major

Robert Maki

Carlo Maley

Reza Malezadeh
Hassan Z Malik

Shakun Malik

Elizabeth Mallon

Marcos Malumbres

Ferdinando Mannello

David Mansfield

Luigi Mansi

Wasat Mansoor

David Mansouri

Zhiyong Mao

Mirko Marabese

Anthony Maraveyas

Faraz K Mardakheh

Coert Margadant

Brigitte Marian

Camille Maringe

Adrian Marino-Enriquez

Maurie Markman

Laura AV Marlow

Maria Marples

Aileen Marshall

Ernest Marshall

Oscar Martínez-Tirado

Richard M Martin

Elisabeth D Martinez

Pablo Martinez Rodriguez

Sandra F Martins

Tanimola Olanrewaju Martins

Joel B Mason

Athena Matakidou

Andres Matoso

Noriomi Matsumura

Keitaro Matsuo

Felicity E B May

Lindsey D Mayo

Xavier Mayol

Vincenzo Mazzaferro

Andrea Mazzarello

Úna C Mc Menamin

Elaine McCartney

W Glenn McCluggage

David McDermott

David McIntosh

Pamela McKay

Ruth F McKee

Andrew McMillan

Donald C McMillan

Mairead Geraldine McNamara

Iain A McNeish

Robert McWilliams

Karen Meaburn

Michaela Medová

Patrick Mehlen

Jan Joseph Melenhorst

Davide Melisi

Begoña Mellado

Javier A Menendez

Usha Menon

Alexander M Menzies

Chris Metcalfe

Michael Michael

Martin Michaelis

Dominique S Michaud

Caroline Michie

Patrick Micke

Mark R Middleton

Kenneth A Miles

Linda R Mileshkin

Anthony B Miller

Anne M Mills
Roger Milne

Koshi Mimori

Junxia Min

Seigo Minami

Toshinari Minamoto

Giuseppe Minniti

Paul S Mischel

Michel Mittelbronn

Eiji Miyoshi

Dominik Modest

Maximilian Moergel

Khalid S Mohammad

Alexander Molassiotis

Damian J Mole

Esther Molina-Montes

Florence Molinie

Richard Molloy

Catherine Monnot

Kristine R Monroe

Gregory Raymond Monteith

Ejun Moon

Sing Yu Moorcraft

Helen Moore

Alessandro Morabito

Irina Mordukhovich

Paolo Morgagni

Carys Morgan

Maria P Morgan

Peter R Morgan

David Morris

Patrick G Morris

David Morrison

Giuseppe Moscelli

Marsha A Moses

Berit Jul Mosgaarde

Elahe Mostaghel

Nicolas Mottet

Judd W Moul

Aristidis Moustakas

Thomas Muerdter

Franco Muggia

Colin R Muirhead

Somnath Mukherjee

Hasan Mukhtar

Volkmar Muller

Erik Munson

Vinayak Muralidhar

Masaaki Muramatsu

Peter Murchie

John Murphy

Brion W Murray

Graeme Murray

Satu Mustjoki

Anthony Mutsears

Christina M Nagle

Vinayak K Nahar

Lillian M Nail

Nita S Nair

Norifumi Naka

Atsushi Nakajima

Margherita Nannini

David Nanus

Steven A Narod

$S$ David Nathenson

Andrea Necchi

Andy R Ness

Nina Neuhaus

Cindy Neuzillet

Patrick Neven

Daotai Nie 
Hans Nijman

Marina Nikiforova

Andrew B Nixon

Simon Noble

Niro Nohata

Anju Nohria

Igor Novitzky-Basso

Stefan Nowicki

Fiona Nussey

Matthew J Nyflot

Karin Nylander

Lennarth Nystrom

Katie O'Brien

James PB O'Connor

Timothy R O'Connor

Norma O'Donovan

Patrick O'Dwyer

Peter O'Dwyer

Eileen Mary O’Reilly

Joyce O'Shaughnessy

Alberto Ocana

Kevin C Oeffinger

Manfred Ogris

Shinya Ohashi

Takahiro Oike

Cristoph Oing

Robert Olson

Adedayo Onitilo

Sjoukje F Oosting

Jose Luis Ordóñez

Steffen Ormanns

David Ornelles

Torben F Ørntoft

Michael Orth

Jennifer Ose

Piet Ost

SJ Otto

Hidetatsu Outani

Mustafa Ozen

Simon Pacey

Oxana Palesh

Daniel H Palmer

Orestis Panagiotou

John Carl Panetta

Klaus Pantel

Nickolas Papadopoulos

Athanasios G Papavassiliou

George Papaxoinis

Ulrich-Frank Pape

Angelo Virgilio Paradiso

Christos Paraskeva

Jong-Wan Park

So Yeon Park

Alexander Parker

Donald Maxwell Parkin

Rowan W Parks

Maria Parpinel

Peter Parsons

Ann H Partridge

Eddy Pasquier

Harvey I Pass

Antonio Passaro

Alpa Patel

Gargi Patel

Atanu Kumar Pati

Santosh Kumar Patnaik

P Patrignani

Mick Peake

Luiz O Penalva

Trevor Penning
George Pentheroudakis

Anita Peoples

Paola Perego

Ethel R Pereira

Lauren C Peres

Aurora Perez-Cornago

Maurice Perol

Godefridus J Peters

Emmanuel F Petricoin III

Paul D Pharoah

Philip A Philip

M Philpott

Werner J Pichler

Dawid Pieper

Ymera Pignochino

LE Pinkerton

Ross Pinkerton

Paul F Pinsky

Alexandros Pintzas

Xavier Pivot

Barry Pizer

L Planelles

Steven C Plaxe

Mary C Playdon

Elizabeth Ruth Plummer

Teresa Pollicino

Sanjay Popat

Irinel Popescu

Cornel Popovici

Richard Possemato

Desmond George Powe

Jenny N Poynter

Friedrich Prall

Aleix Prat

Hans Prenen

Michael F Press

Timothy J Price

Holly G Prigerson

Elena-Sophie Prigge

John N Primrose

Robert M Prins

Mark Pritchard

Carol Prives

David Propper

Sylvain Provot

Giancarlo Pruneri

Ioannis Psallidas

Florian Puls

SA Purcell

Colin A Purdie

Bin-Zhi Qian

Halima Quadid-Ahidouch

Gaelle Quereux

JM Quillin

James M Rae

Kunal Rai

Krishnaraj Rajalingam

Suresh S Ramalingam

Ramesh K Ramanathan

Sheela Rao

Martine F Raphael

Ardeshir R Rastinehad

AD Raval

Nicholas Simon Reed

Myrddin Rees

Helen Louise Reeves

Scott Regenbogen

Alison Reid

Andrew G Renehan

Lindsay A Renfro
Michele Reni

Gad Rennert

Caroline Reuter

HT Reyburn

Anna K.L Reyners

Andrew R Reynolds

Kyle A Richards

Ellen Richmond

Alberto Righi

Alistair E Ring

Mauro Risio

Kathryn Robb

Karin Roberg

Max Robinson

Stuart Michael Robinson

Mark E Robson

Sonia Rocha

Gary Rodin

Rene Rodriguez

Sandra Rodriguez-Perales

Simon Rogers

Theodore Rokkas

Eve Roman

Matti A Rookus

Michael Rose

Rafael Rosell

Robert Rosenberg

Antonia K Roseweir

Paul J Ross

Richard Ross

Sacha I Rothschild

David Rowley

Campbell SD Roxburgh

Sinchita Roy-Chowdhuri

Greg Rubin

Avima M Ruder

Simon Rule

Antonio Russo

Alison S Rustagi

Gordon JS Rustin

Mark J Rutherford

Joan Sabaté

Joseph Sacco

Angelica A Saetta

Satoru Sagae

Fabiano P Saggioro

Pipsa Saharinen

Deepak Sahasrabudhe

Nagahiro Saijo

Noriaki Sakuragi

Ritu Salani

Ramon Salazar

Roberto Salgado

David A Sallman

Leonard B Saltz

Sujith Samarasinghe

Wolfram Samlowski

John Sampson

Alessandro D Santin

Daniele Santini

Giorgio Santoni

Yasutsuna Sasaki

Toyomi Satoh

Prakash Satwani

Nicolai E Savaskan

Nadia Sawicka-Gutaj

Mario Scartozzi

Sebastian Schölch

Zachary T Schafer

Lars M Schiffmann
Daniela Schmid

Marjanka K Schmidt

Mia Schmidt-Hansen

Martin Schneider

Minouk J Schoemaker

Matthias B Schulze

Thomas Schweiger

Jochen M Schwenk

Lukas Schwingshackl

Jean-Yves Scoazec

Mark Sculpher

Catherine R Sears

Lai-Chu See

John Paul Seenan

E Segelov

Georg Seifert

Abhijit Sen

Consolato Sergi

Guido Serini

Manuel Serrano

Bryan Serrels

Cristiana Sessa

Gautam Sethi

Takashi Seto

Elena Genevieve Seviour

David B Shackelford

Mark Shackleton

Mazyar Shadman

Maitri Yogen Shah

Jonathan Shamash

Rohini Sharma

Kieran Sheahan

Feng Shen

Patrick Shenjere

Frances Shepherd

Tatsuhiro Shibata

Anthony F Shields

Hiroaki Shiina

Joanna Shim

Yohei Shimono

Stacey Shiovitz

Janet Shipley

Mamoru Shoji

SV Shrikhande

Jiang Shudong

Angels Sierra

Alice E Simon

Michael Simon

Narendra Singh

Heath D Skinner

Susan L Slager

Brian M Slomovitz

Emily Slotkin

Cathy Smith

Elizabeth C Smyth

Matthew John Soeberg

Huan Song

Mingyang Song

Gabe S Sonke

Makoto Sonobe

Guru Sonpavde

Pierre Sonveaux

Prasanna Sooriakumaran

Anna Sorace

Boe Sandahl Sorensen

Poul HB Sorensen

Robert Soslow

Khalid Sossey-Alaoui

Pierre Soubeyran

Nuno Sousa 
Giulio Spagnoli

Helen Speedy

Valerie Speirs

James F Spicer

Salvatore Spicuglia

Ashok Srinivasan

Johan Staaf

Sasha E Stanton

Naureen Starling

Par Stattin

Justin Stebbing

Susan E Steck

Michael Steffens

Stacey M Stein

Alexander Steinle

Arnulf Stenzl

Victoria Stevens

Douglas Stewart

Sebastian Stintzing

Karyn Stitzenberg

Jon Stobo

Nikolas Hendrik Stoecklein

Sandro J Stoeckli

Rachael Stolzenberg-Solomon

Emily Stone

Dawn Storey

John Strickler

Jonathan R Strosberg

Robin Stuart-Harris

Amit Sud

David John Sugarbaker

SY Suh

Frank Sullivan

Richard Sullivan

Lei-min Sun

Xuezheng Sun

Arthur Sun Myint

Y Suzuki

Magali Svrcek

John Swanson

John Sweetenham

Anthony J Swerdlow

Stefan Nicholas Symeonides

Lazlo Tabar

Lee-Hwa Tai

Diana Tait

Valerie Taly

Rulla M Tamimi

David Tan

Noriko Tanaka

Shinji Tanaka

Toshiyasu Taniguchi

Ian F Tannock

Jose Tanus-Santos

Menghua Tao

Coya Tapia

Natasha Tasevska

Riccardo Taulli

Susan L Teitelbaum

Natercia Teixeira

Arnoud Templeton

Daniel A Tennant

Ioannis Tentes

Masanori Terashima

Vinay Tergaonkar

Kathryn L Terry
May-Britt Tessem

Giorgio Tettamanti

Ho Thai

Bijin Thajudeen

Evropi Theodoratou

Fiona Thistlethwaite

Anne Thomas

Jeremy Thomas

Jeremy St J Thomas

Laura E Thomas

Patricia Thompson-Carino

C A Thomson

Melissa SY Thong

Gavin Thurston

Mark Thursz

Shruti Rakesh Tiwari

Yoshihiko Tomita

Ian PM Tomlinson

Massimo Tommasino

Sarah Tonack

Betty C Tong

Johanna Torfadottir

K Tornquist

Davis Y Torrejon

Tibor Tot

Hidenori Toyoda

Ly Thi Hai Tran

Gerd Trano

Lois B Travis

Antonia Trichopoulou

Daniel Trifiletti

Quoc-Dien Trinh

Ngan-Ming Tsang

Jun-Chieh Tsay

Konstantinos Tsilidis

Pelagia G Tsoutsou

G Tuccari

Paola Turano

Baris Ismail Turkbey

Richard C Turkington

Michelle Turner

Jeremy Udkoff

Hideki Ueno

Danny Ulahannandan

Jane Ussher

Claire M Vajdic

Lil Valentin

Nicola Valeri

Erin Van Blarigan

Mieke van Bockstal

Peter A van Dam

Matthijs van de Rijn

Thierry Van den Bosch

Winette TA van der Graaf

Michiel S van der Heijden

Anthonie J van der Wekken

Dik C van Gent

Richard van Hillegersberg

Jean-Luc Van Laethem

Jan van Meerbeeck

Nicolien van Ravesteyn

Jacco van Rheenen

Peter SN van Rossum

Ari Vanderwalde

Sakari Vanharanta

Angelo Vanzulli
Christine Varon

Chenthamarakshan Vasu

Naveen S Vasudev

Lars J Vatten

Ines Vaz-Luis

Peter Vedsted

Nienke J Veldhuijzen

Galina Velikova

Mary Anna Venneri

Freija Verdoodt

Helena M Verkooijen

SK Verma

Louis Vermeulen

Antonella Verrienti

Marco Vinceti

Charlotte M Vines

Susanne Vinkel Koch

Amaya Viros

Sabine Vogler

Stefano Volinia

Jon Wadsley

Anna-Dorothea Wagner

Zev A Wainberg

Richard Wakeford

Heather A Wakelee

CJ Wakeman

marjorie walker

Lucy Wall

Fiona M Walter

Jun Wan

Christina Wang

Lai Mun Wang

Liwei Wang

Lynn Wang

Mengzhao Wang

Rong Wang

Ying Wang

Zhenning Wang

Erica T Warner

Harpreet Wasan

James Wason

Justin Waters

David Watkins

Alastair Watson

David G Watt

Erin Wei

Wilko Weichert

Robin A Weiss

Wei Wen

Priya Werahera

Shannon Westin

Kenneth Westover

Alexandra J White

Emily White

Jeff D White

W Ruprecht Wiedemeyer

Lisa Wiesmueller

Simon Wigfield

Paul Wilkerson

Christopher G Willett

Gareth Haydn Williams

Grant R Williams

William R Wilson

Danyelle Winchester

Marcy Winget

G Bea A Wisman
Ken Witwer

Isabell Witzel

Penella J Woll

Lennie Wong

Li F Wong

Victoria Woodcock

Ian Woolhouse

Margaret Wrensch

Kusheng $\mathrm{Wu}$

Olivia Wu

Sherry Y Wu

Shu-Jie Xia

Shunbin Xiong

Rui-hua Xu

Tomonori Yaguchi

E Yakirevich

Sohsuke Yamada

Ryuya Yamanaka

Eric $\mathrm{H}$ Yang

Hushan Yang

Pan-Chyr Yang

Song Yao

Timothy A Yap

Clayton Yates

Weimin Ye

JJ Yeh

Sai Yendamuri

Belinda Yeo

Sai-Ching J Yeung

Min Yi

John Yim

Kein-Leong Yim

Yanping Ying

Kent Yip

Yoshihito Yokoyama

Kazuhiro Yoshida

Greg Yothers

Teresa Young

Stacey Young-McCaughan

George M Yousef

Qian Z

Sophia Zackrisson

Anna J Zaczek

Pauline Zaenker

Per-Henrik Zahl

Charles Zaloudek

Rita Zamarchi

Bianca Maria Zani

C Zani

Marjorie G Zauderer

AG Zeimet

Tai-Ping Zhang

Xiaowen Zhang

Jianhua Zhao

Limin Zheng

Pengcheng Zhu

Richard Zigeuner

Inti Zlobec

Michal A Zmijewski

Hui Zong

Antonella Zucchetto

István Zupkó

Axel zur Hausen

Jeff I Zwicker 